\title{
Distress and Prevention Behaviors During the COVID-19 Pandemic Among College Students: the Moderating Role of Resilience
}

\author{
Tyler A. Kuhn ${ }^{1}(0)$. Anthony Vander Horst ${ }^{2}$. Gregory C. Gibson ${ }^{2} \cdot$ Kimberly A. Cleveland $^{3} \cdot$ Craig Wawrosch $^{2}$. \\ Cynthia Hunt $^{2}$ - Christopher J. Woolverton ${ }^{4}$. Joel W. Hughes ${ }^{1}$
}

Accepted: 3 October 2021 / Published online: 12 October 2021

(c) International Society of Behavioral Medicine 2021

\begin{abstract}
Background Pandemics can generate considerable distress, which can affect prevention behaviors. Resilience may buffer the negative effects of distress on engagement in relevant prevention behaviors, which may also hold true for COVID-19 prevention behaviors. The objective of the current study was to evaluate whether resilience moderated the relationship between distress and COVID-19 prevention behaviors early in the pandemic.

Methods Data were collected via surveys in which all students at a large midwestern university were emailed invitations beginning March 18, 2020. Surveys were completed by 5,530 individuals. In addition to demographic questions and items about COVID-19 prevention behaviors, distress was assessed using the K6 Distress Scale and resilience using the Brief Resilience Scale. Data were analyzed using moderator regression analysis.

Results Resilience moderates the effects from distress to prevention behaviors, such that the relationship was stronger for individuals with higher resilience than for individuals with lower resilience. When resilience was one standard deviation below the mean, at the mean value of resilience, and when resilience was one standard deviation above the mean, there was a significant positive relationship between distress and COVID-19 prevention behaviors. However, the relationship was strongest for those with high resilience, and lowest for those with low resilience.

Conclusions In the current sample, resilience appeared to influence the strength of the relationship between distress and COVID-19 prevention behaviors. Having higher resilience may promote positive adaptation to distress, leading individuals to engage in a greater number of disease-related prevention behaviors. Future research should examine this relationship longitudinally and in relation to differing constructs of resilience.
\end{abstract}

\section{Introduction}

Among other stressors, the COVID-19 pandemic has led to feelings of uncertainty, social isolation, fear that one or one's family may become ill, and fear about one's finances [1]. Together, these stressors associated with the outbreak have led to a significant increase in reported distress [2]. Distress can lead to many negative outcomes, but one important

Tyler A. Kuhn

tkuhn6@kent.edu

1 Department of Psychological Sciences, Kent State University, P.O. Box 5190, Kent, OH 44242, USA

2 Department of Sociology, Kent State University, Kent, $\mathrm{OH} 44242$, USA

3 College of Nursing, Kent State University, Kent, OH 44242 , USA

4 Department of Epidemiology, Kent State University, Kent, OH 44242, USA consideration is how distress may influence individual prevention behaviors, especially those related to the pandemic.

Widespread behavior change is effective in lessening disease transmission [3, 4]. Among other behaviors, wearing a mask in public, quarantining while sick, and avoiding large gatherings have shown to mitigate disease transmission. These strategies are especially important while pharmacological preventative interventions are not available and are recommended by the Centers for Disease Control and Prevention [4]. However, the adoption of prevention behaviors is not global [5].

While generally accepted that distress may lead to maladaptation including decreased engagement in health behaviors, research suggests that increased anxiety and distress may motivate individuals to engage in prevention behaviors. During a disease outbreak, distress has been associated with increased adherence to behavioral recommendations to prevent negative disease outcomes. This has been shown through research conducted during both the H1N1 ("swine flu") and SARS-CoV epidemics [6, 7]. 
Another construct relevant to both distress and disease prevention behaviors is resilience, which may generally mean bouncing back from stress or positively recovering from stress [8]. Dispositional resilience buffers the negative effects of distress on engagement in general health behaviors [9-11]. Historically, resilience has been shown to moderate the relationship between distress and dietary habits [9]. During the COVID-19 pandemic, research so far suggests that resilience moderates the relationship between distress and psychological functioning and eating habits [10, 11]. However, resilience can additionally be defined as promoting positive adaptation or flourishing throughout adversity. In situations where distress may have a negative effect on unhealthy behaviors (e.g., eating psychopathology), resilience might buffer the negative effect of distress on behaviors [9-11]. However, when distress is hypothesized to promote increased engagement in healthy behaviors (e.g., disease prevention behaviors), resilience may function to enhance the positive effect distress has on engagement in healthy behaviors [12,13]. For preventative behaviors, distress may promote increased adherence. It is possible that higher resilience might promote increased engagement in preventative behaviors at each level of distress.

College students may be particularly vulnerable to increased distress and subsequent changes in prevention behaviors due to the COVID-19 pandemic. Changes at colleges and universities have led to increased distress, including financial stress, uncertainty around living situations, having less access to mental health care, and concerns about graduation and internship changes [14]. Knowing that a college community may be at high risk of increased distress, it is important to understand factors that may help promote increased prevention behaviors during this time, one of which might be resilience.

After the World Health Organization declared the COVID19 outbreak a pandemic on March 11, 2020, we distributed surveys using protocols previously developed and piloted during the Ebola Virus Disease (EVD) epidemic in 2014 to examine COVID-19 distress in a university community [15]. The current aim was to determine whether resilience would moderate the relationship between distress and COVID-19 prevention behaviors during the pandemic. It was hypothesized that high resilience would be related to higher engagement in COVID-19 prevention behaviors across levels of distress.

\section{Methods}

\section{Demographic Information}

The current study was a cross-sectional design utilizing a convenient sample of college students. All students at a large midwestern university system were emailed invitation links to complete an internet survey (Qualtrics ${ }^{\mathrm{XM}}$, https:// www.qualtrics.com/) with recruitment beginning on March 18, 2020, and ending on April 15th, 2020. The total survey response rate among undergraduate and graduate students was $17.8 \%$ with a total of 5,547 (4148 undergraduate) completed student surveys that passed a validity check (e.g., "Choose 4 for this item") out of 30,996 distributed surveys. There was no incentive for participation. Participants were included if they were either an undergraduate or graduate student and were 18 years old or older. The survey included demographic items as well as questions related to distress, resilience, and COVID-19 prevention behaviors. A total of 5424 participants had completed ratings for distress, resilience, and COVID-19 prevention behaviors and were included in the current study. The institutional review board at a midwestern university approved all data collection procedures (IRB-protocol \#20-145).

\section{Measures}

Distress The Kessler Psychological Distress Scale (K6) was used to measure distress $[16,17]$. The $\mathrm{K} 6$ is a widely used 6-item self-report measure of nonspecific distress and prompts individuals to rate how often they have experienced symptoms such as "nervous," "hopeless," or "restless or fidgety" over the previous month on a 5-point Likert scale with 1 indicating "none of the time," and 5 indicating "all of the time." Scores on the K6 are summed together and range from 0 to 24 , with a score between 5 and 12 indicating moderately elevated distress and a score of 13 or above indicating severe distress. The K6 has robust psychometric properties within adult populations, ranging across multiple languages. Within the current sample, the K6 had strong internal consistency $(\alpha=0.86)$.

Resilience The Brief Resilience Scale (BRS) was used to measure resilience [8]. The BRS is a brief, 6-item, selfreport measure of an individual's ability to "bounce back" from stress. Three items are positively worded and three are negatively worded. When scored, the negatively worded items are reversed scored and a total is computed. Individuals are asked on a 5-point scale how much they disagree or agree with statements such as, "it does not take me long to recover from a stressful event." Internal consistency for the BRS was high in the current sample $(\alpha=0.88)$.

COVID-19 Prevention Behaviors COVID-19 prevention behaviors were measured via a series of 12 yes/no survey items. Study participants were asked whether or not they avoid those who cough/sneeze, avoid large gatherings, wash their hands/use hand sanitizer frequently, avoid public transportation/school/work, make sure to get adequate sleep, cover coughs/sneezes, and canceled travel plans outside the USA/inside the USA outside Ohio/inside Ohio. Items were either scores as 0 for not engaging in the behavior or as 1 for engaging in the behavior. "Yes" responses (those marked as 
1) were summed together to produce a summary COVID19 prevention behaviors score. Of note, at the time of data collection, using a face mask was only recommended for individuals experiencing COVID-19 symptoms and not yet for widespread population adoption and as such was not included in the analysis [18]. Internal consistency for the COVID-19 prevention behaviors scale used was $\alpha=0.68$ in the current sample.

Demographic Characteristics The study survey also included important demographic items of age, sex, and race.

\section{Regression and Moderation Analysis}

Sample baseline characteristics were characterized as means (standard deviations) and percent (frequencies). Data was first screened for missing data, outliers, and normality. To test for outliers, a three-standard deviation from the mean cut-off was utilized. For normalcy, a 1.96 standard deviation from the mean cut-off was utilized. There were no missing data or outliers, and normalcy was confirmed for all variables. To test the moderating effect of resilience on the association between distress and COVID-19 prevention behaviors, a moderation analysis was conducted using the PROCESS macro (model 1) produced by Hayes for IBMSPSS version 27.0 statistical software. Hayes's PROCESS macro computes multiple ordinary least squares regressions, which include the centered product term representing the interaction between distress and resilience as a predictor of COVID-19 prevention behaviors. Reported effect estimations were unstandardized regression coefficients with statistical significance set at 0.05 . Controlled for covariates in this analysis included age, sex, and race. Simple slope analysis was conducted using the mean and $+/-1$ standard deviation from the mean.
Table 1 Characteristics of participants $(n=5424)$

\begin{tabular}{|c|c|c|}
\hline & & $M(S D)$ or $n(\%)^{\mathrm{a}}$ \\
\hline \multicolumn{3}{|l|}{ Demographic and medical factors } \\
\hline \multicolumn{3}{|l|}{ Age range } \\
\hline $18-19$ & $1081(19.90)$ & \\
\hline $20-24$ & $2641(48.70)$ & \\
\hline $25-29$ & $652(12.0)$ & \\
\hline $30-34$ & $372(6.90)$ & \\
\hline $35-39$ & $218(4.00)$ & \\
\hline $40-44$ & $156(2.90)$ & \\
\hline $45-49$ & $145(2.70)$ & \\
\hline $50-54$ & $83(1.50)$ & \\
\hline $55-59$ & $49(.90)$ & \\
\hline $60-64$ & $16(.30)$ & \\
\hline $65-69$ & $7(.10)$ & \\
\hline $70-74$ & $2(.00)$ & \\
\hline $75+$ & $2(.00)$ & \\
\hline \multicolumn{3}{|l|}{ Sex } \\
\hline Female & $4014(74.00)$ & \\
\hline Male & $1410(26.00)$ & \\
\hline \multicolumn{3}{|l|}{ Race } \\
\hline American Indian/Alaska native & $12(.20)$ & \\
\hline Asian (includes Indian subcontinent) & $261(4.80)$ & \\
\hline Black or African American & $274(5.10)$ & \\
\hline Native Hawaiian or other Pacific Islander & $2(.00)$ & \\
\hline White & $4720(87.00)$ & \\
\hline Other & $155(2.90)$ & \\
\hline Undergraduate student (compared to graduate student) & $4148(76.50)$ & \\
\hline Kessler Psychological Distress Scale (K6) & $14.16(5.66)$ & \\
\hline Brief Resilience Scale (BRS) & $20.38(4.81)$ & \\
\hline COVID-19 prevention behaviors & $9.10(2.36)$ & \\
\hline
\end{tabular}

${ }^{a}$ For continuous variables, means and standard deviations are reported. For categorical variables, sample size and percentages are reported 
Table 2 Bivariate correlations for study variables
1

1. Age

2. Sex

3. Race

4. Kessler Psychological Distress Scale (K6)

5. Brief Resilience Scale (BRS)

6. COVID-19 prevention behaviors

${ }^{*} p<.05 ;{ }^{* *} p<.01$
2

3

$-.02$

$-.03 * \quad .02$

$-.17 * * \quad .15^{* *}$

$.20 * * \quad-.14 * *$

$.12 * *$

$.11 * *$

.01

.02

$-.51 * * \quad-$

$-.09 * *$

\section{Results}

\section{Participants}

The current study sample included predominantly young (48.70\% aged $20-24)$, white (87\%), females (74\%). The average K6 score was $14.16(S D=5.66)$, indicting a severe level of distress. The average BRS was $20.38(S D=4.81)$, while the average number of COVID-19 prevention behaviors engaged in was $9.10(S D=2.36)$. Further sample characteristics are shown in Table 1. For bivariate correlations among study variables, see Table 2 .

\section{Moderation Analysis}

Resilience was examined as a moderator of the relation between distress and COVID-19 prevention behaviors. For COVID-19 prevention behaviors, the overall model was statistically significant, $R^{2}=0.03, F(6,5416)=22.21$, $p<0.001$. Controlling for age, sex, and race, the interaction of resilience and distress explained a significant increase in variance in prevention behaviors, $\Delta R^{2}=0.001, F(1$, $5416)=5.37, p<0.05$ (see Table 3).

When resilience was one standard deviation below the mean, there was a significant positive relationship between distress and COVID-19 prevention behaviors, $b=0.03$, $95 \%$ CI $[0.01,0.04], t=3.13, p<0.01$. At the mean value of resilience, there was a significant positive relationship between distress and COVID-19 prevention behaviors, $b=0.04,95 \%$ CI [0.03, 0.0.5], $t=5.37, p<0.001$. When resilience was one standard deviation above the mean, there was a significant positive relationship between distress and COVID-19 prevention behaviors, $b=0.05,95 \%$ CI [0.03, 0.07], $t=5.03, p<0.001$. In total, resilience moderated the relationship between distress and COVID19 prevention behaviors, such that the relationship was stronger for individuals with higher resilience than for individuals with lower resilience. For simple slopes, see Fig. 1.

\section{Discussion}

The current investigation examined whether resilience moderated the effect of distress on COVID-19 prevention behaviors among a sample of college students during the early days of the COVID-19 pandemic. In the current sample, resilience moderated the relationship between distress and COVID-19 prevention behaviors, in that distress was more strongly associated with COVID-19 prevention behaviors in students higher in resilience.

The results are consistent with literature on resilience, distress, and health behaviors, in that resilience improved engagement in health behaviors $[10,11]$. The novelty of the current investigation is in suggesting that resilience moderates the positive association between distress and prevention behaviors, unlike previous literature that only examined health behaviors unrelated to prevention specifically. At higher levels of distress, higher self-reported ability to bounce back from stress may have prompted additional positive adaptation as a response to mounting distress.

Table 3 Linear model of predictors of COVID-19 prevention behaviors

\begin{tabular}{lllll}
\hline & $b$ & SE B $t$ & $p$ \\
\hline Constant & 8.83 & .23 & 38.10 & $p<.001$ \\
& {$[8.38,9.29]$} & & & \\
Psychological distress & .04 & .01 & 5.37 & $p<.001$ \\
$\quad$ (centered) & {$[.03, .05]$} & & & \\
$\begin{array}{l}\text { Psychological resilience } \\
\quad \text { centered) }\end{array}$ & .01 & .01 & 1.38 & $p=.17$ \\
Psychological distress $\times$ & {$[-.01, .03]$} & & & \\
psychological resilience & .003 & .001 & 2.32 & $p<.05$ \\
Age & {$[.00, .01]$} & & & \\
& -.01 & .02 & -.45 & $p=.66$ \\
Sex & {$[-.05, .03]$} & & & \\
& .64 & .08 & 8.17 & $p<.001$ \\
Race & {$[.50, .80]$} & & & \\
& -.16 & .04 & -4.36 & $p<.001$ \\
& {$[-.24,-.10]$} & & & \\
\hline
\end{tabular}

$R^{2}=.03 . \Delta R^{2}=.001, p<.05$ 
Fig. 1 Simple slopes

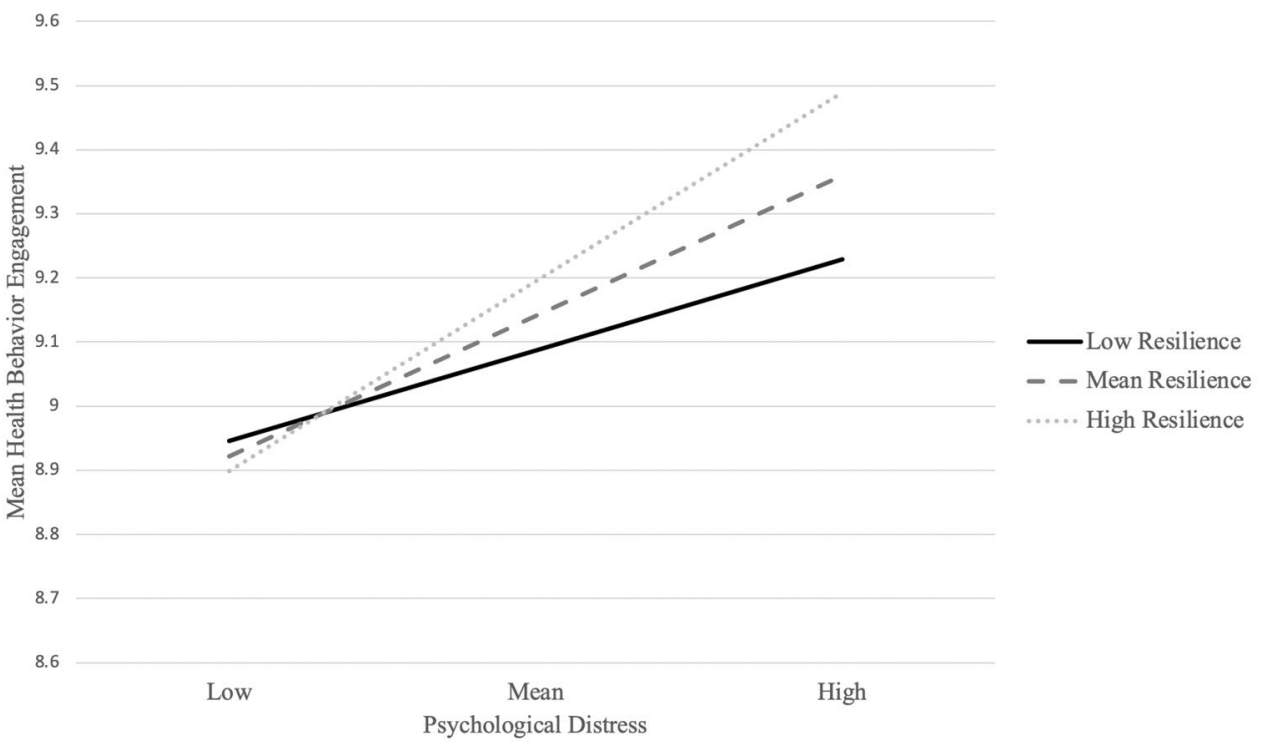

The present findings have several clinical and research implications. Mostly, results of this investigation suggest that level of resilience may play an adaptive role in the relationship between distress and COVID-19 prevention behaviors. On a larger scale, resilient students may be more likely to engage in prevention behaviors in the wake of mounting distress. Clinically, resilience interventions could be implemented at the university level, such as "Battle Buddies" a resiliency intervention developed by the United States Army, and more recently successfully utilized by some health care networks during the COVID19 pandemic [19]. Overall, continued examination of how protective factors, such as resilience, influence the effects of distress may be a viable avenue for promoting positive behavioral outcomes.

This study had several strengths. The sampling period was very early in the COVID-19 pandemic, beginning the week after all instruction moved to remote delivery and before the statewide stay at home order was issued on March 22, 2020. When recruiting began, there were no reported cases of COVID-19 in the university community, and yet distress among students was already very high [20]. The survey response rate was high for an uncompensated survey of all students, which resulted in a large sample. Finally, we were able to evaluate the interaction of resilience and distress as the pandemic unfolded locally, precisely when widespread adoption of prevention behaviors was the only viable approach to containing the spread of the novel coronavirus.

Limitations of the current study should also be noted. First, a cross-sectional, convenience sample of college students may make generalizability difficult. The response rate was also relatively low (17.8\%). More so, the COVID-19 prevention behaviors self-report measure used in this study may be limited. Self-report measures of behavior can be subject to reporter bias. The measure also only examines twelve possible COVID-19 prevention behaviors deemed important during the early stages of the COVID-19 pandemic. Also, some stressors associated with the COVID-19 pandemic might contribute to a higher level of distress but promote preventive behaviors in college students (e.g., switching to online classes might not only increase distress but also promote healthy prevention behaviors). Future research should consider such variables. Additionally, the variance explained for by the interaction between distress and resilience was small. This may be due to a ceiling effect for the measure of COVID-19 prevention behaviors, which might have attenuated the size of the effect that could be detected. This also could be an attribute of using nonspecific/simple measures such as the K6 and BRS while studying a specific COVID19 context. This may hinder the overall clinical utility of the current results. However, when testing hypotheses, even a small change in variance explained in the expected direction is supportive of the theory being evaluated.

\section{Conclusion}

In summary, here, it is reported that resilience moderates the relationship between distress and COVID-19 prevention behaviors among a sample of college students. As distress increased, higher resilience was related to increased COVID19 prevention behaviors. Having higher resilience may promote positive adaptation to distress, leading individuals to engage in a greater number of disease-related prevention behaviors. Future research should examine this relationship longitudinally and in relation to differing constructs of resilience. Additionally, future research should consider other potential moderators such as psychological flexibility, 
a variable related to resilience. However, if replicated, our findings suggest that promoting greater individual resilience may promote better engagement in disease-related prevention behaviors in the wake of mounting distress.

\section{Declarations}

Ethical Approval All procedures performed in studies involving human participants were in accordance with the ethical standards of the institutional research committee and with the 1964 Helsinki declaration and its later amendments or comparable ethical standards.

Informed Consent Informed consent was obtained from all individual participants included in the study.

Conflict of Interest The authors declare no competing interests.

\section{References}

1. Reger M, Stanley I, Joiner, T. Suicide mortality and coronavirus disease 2019-a perfect storm?. JAMA psychiatry. 2020.

2. Wang C, Pan R, Wan X, et al. Immediate psychological responses and associated factors during the initial stage of the 2019 coronavirus disease (COVID-19) epidemic among the general population in China. International journal of environmental research and public health. 2020;17(5):1729.

3. Centers for Disease Control and Prevention. "Communities, schools, workplaces, \& events." https://www.cdc.gov/coronavirus/ 2019-ncov/community/community-mitigation.html. Accessed 8 Jan 2021.

4. Centers for Disease Control and Prevention. Social Distancing https://www.cdc.gov/coronavirus/2019-ncov/community/ community-mitigation.html. Accessed 8 Jan 2021.

5. Czeisler M, Tynan M, Howard M, et al. Public attitudes, behaviors, and beliefs related to COVID-19, stay-at-home orders, nonessential business closures, and public health guidance-United States, New York City, and Los Angeles, May 5-12, 2020. Morb Mortal Wkly Rep. 2020;69(24):751.

6. Leung G, Ho L, Chan S, et al. Longitudinal assessment of community psychobehavioral responses during and after the 2003 outbreak of severe acute respiratory syndrome in Hong Kong. Clin Infect Dis. 2005;40(12):1713-20.

7. Rubin G, Amlôt R, Page L, et al. Public perceptions, anxiety, and behaviour change in relation to the swine flu outbreak: cross sectional telephone survey. Bmj. 2009;339:b2651.
8. Smith B, Dalen J, Wiggins K, et al. The brief resilience scale: assessing the ability to bounce back. Int J Behav Med. 2008;15(3): 194-200.

9. Thurston I, Hardin R, Kamody R, Herbozo S, Kaufman C. The moderating role of resilience on the relationship between perceived stress and binge eating symptoms among young adult women. Eat Behav. 2018;29:114-9.

10. Kavčič T, Avsec A, Zager KG. Psychological functioning of Slovene adults during the COVID-19 pandemic: does resilience matter? Psychiatr Q. 2020;92(1):207-16.

11. Du C, Zan M, Cho M, et al. The effects of sleep quality and resilience on perceived stress, dietary behaviors, and alcohol misuse: a mediation-moderation analysis of higher education students from Asia, Europe, and North America during the COVID-19 pandemic. Nutrients. 2021;13(2):442.

12. Southwick SM, Bonanno GA, Masten AS, Panter-Brick C, Yehuda R. Resilience definitions, theory, and challenges: interdisciplinary perspectives. Eur J Psychotraumatol. 2014;5(1):25338.

13. Tusaie K, Dyer J. Resilience: a historical review of the construct. Holist Nurs Pract. 2004;18(1):3-10.

14. American College Health Association. (2020). The impact of COVID-19 on college student well-being. American College Health Association. Retrieved from https://www.acha.org/documents/ncha/ Healthy_Minds_NCHA_COVID_Survey_Report_FINAL.pdf

15. Shook E, Curtis A, Curtis J, et al. Assessing the geographic context of risk perception and behavioral response to potential ebola exposure. Int J Environ Res Public Health. 2019;16(5):831.

16. Kessler R, Baker P, Colpe L, et al. Screening for serious mental illness in the general population. Arch Gen Psychiatry. 2003;60(2):184-9.

17. Kessler R, Green J, Gruber M, et al. Screening for serious mental illness in the general population with the K6 screening scale: results from the WHO World Mental Health (WMH) survey initiative. Int J Methods Psychiatr Res. 2010;19(S1):4-22.

18. Centers for Disease Control and Prevention. "Coronavirus disease 2019 (COVID-19) prevention \& treatment." https://www. cdc.gov/coronavirus/2019-ncov/prevent-getting-sick/prevention. html. Accessed 8 Jan 2021.

19. Albott C, Wozniak J, McGlinch B, Wall M, Gold B, Vinogradov S. Battle buddies: rapid deployment of a psychological resilience intervention for health care workers during the COVID-19 pandemic. Anesth Analg. 2020;131(1):43-54.

20. Hughes J, Vander Horst A, Gibson G, et al. COVID-19 pandemic exacerbates psychological distress of college students. Manuscript Under Review.

Publisher's Note Springer Nature remains neutral with regard to jurisdictional claims in published maps and institutional affiliations. 